\title{
Generalizations to monotonicity for uniform convergence of double sine integrals over $\overline{\mathbb{R}}_{+}^{2}$
}

\author{
by

\section{PÉTer Kórus and Ferenc Móricz (Szeged)}

\begin{abstract}
We investigate the convergence behavior of the family of double sine integrals of the form$$
\int_{0}^{\infty} \int_{0}^{\infty} f(x, y) \sin u x \sin v y d x d y, \quad \text { where } \quad(u, v) \in \mathbb{R}_{+}^{2}:=\mathbb{R}_{+} \times \mathbb{R}_{+},
$$

$\mathbb{R}_{+}:=(0, \infty)$, and $f: \mathbb{R}_{+}^{2} \rightarrow \mathbb{C}$ is a locally absolutely continuous function satisfying certain generalized monotonicity conditions. We give sufficient conditions for the uniform convergence of the remainder integrals $\int_{a_{1}}^{b_{1}} \int_{a_{2}}^{b_{2}}$ to zero in $(u, v) \in \mathbb{R}_{+}^{2}$ as $\max \left\{a_{1}, a_{2}\right\} \rightarrow \infty$ and $b_{j}>a_{j} \geq 0, j=1,2$ (called uniform convergence in the regular sense). This implies the uniform convergence of the partial integrals $\int_{0}^{b_{1}} \int_{0}^{b_{2}}$ in $(u, v) \in \mathbb{R}_{+}^{2}$ as $\min \left\{b_{1}, b_{2}\right\} \rightarrow \infty$ (called uniform convergence in Pringsheim's sense). These sufficient conditions are the best possible in the special case when $f(x, y) \geq 0$.
\end{abstract}

\section{Introduction: Convergence of double integrals over $\overline{\mathbb{R}}_{+}^{2}$. Let} $\phi: \overline{\mathbb{R}}_{+}^{2} \rightarrow \mathbb{C}$ be a locally integrable function over $\overline{\mathbb{R}}_{+}^{2}$ in Lebesgue's sense, in symbols: $\phi \in L_{\text {loc }}^{1}\left(\overline{\mathbb{R}}_{+}^{2}\right)$, where $\overline{\mathbb{R}}_{+}:=[0, \infty)$. By definition, the double integral

$$
\int_{0}^{\infty} \int_{0}^{\infty} \phi(x, y) d x d y
$$

is said to converge in Pringsheim's sense if the partial integrals

$$
I\left(\phi ; b_{1}, b_{2}\right):=\int_{0}^{b_{1}} \int_{0}^{b_{2}} \phi(x, y) d x d y, \quad\left(b_{1}, b_{2}\right) \in \mathbb{R}_{+}^{2},
$$

2010 Mathematics Subject Classification: Primary 26A46, 26B30, 40A10; Secondary 42A32, 42B99.

Key words and phrases: convergence of double integrals over $\overline{\mathbb{R}}_{+}^{2}$ in the regular sense, convergence in Pringsheim's sense, monotonically decreasing functions on $\mathbb{R}_{+}^{2}$, mean value bounded variation functions (MVBVF), non-onesided bounded variation functions (NBVF), family of double sine integrals over $\overline{\mathbb{R}}_{+}^{2}$, uniform convergence. 
converge to a finite limit as $b_{1}, b_{2} \rightarrow \infty$ independently of each other, in symbols: $\min \left\{b_{1}, b_{2}\right\} \rightarrow \infty$.

By the Cauchy convergence criterion, a necessary and sufficient condition for the convergence of the double integral (1.1) in Pringsheim's sense is that for every $\varepsilon>0$ there exists $b_{0}=b_{0}(\varepsilon)$ such that

$$
\left|I\left(\phi ; b_{1}, b_{2}\right)-I\left(\phi ; b_{3}, b_{4}\right)\right|<\varepsilon \text { if } \min \left\{b_{1}, b_{2}, b_{3}, b_{4}\right\}>b_{0} .
$$

It follows from the convergence in Pringsheim's sense that the remainder integrals satisfy

$$
\begin{aligned}
& \int_{a_{1}}^{b_{1}} \int_{a_{2}}^{b_{2}} \phi(x, y) d x d y \\
& =I\left(\phi ; b_{1}, b_{2}\right)-I\left(\phi ; a_{1}, b_{2}\right)-I\left(\phi ; a_{2}, b_{1}\right)+I\left(\phi ; a_{1}, a_{2}\right) \rightarrow 0 \\
& \quad \text { as } \min \left\{a_{1}, a_{2}\right\} \rightarrow \infty, b_{j}>a_{j}>0, j=1,2 .
\end{aligned}
$$

By definition, the double integral (1.1) is said to converge in the regular sense if

$$
\int_{a_{1}}^{b_{1}} \int_{a_{2}}^{b_{2}} \phi(x, y) d x d y \rightarrow 0 \quad \text { as } \max \left\{a_{1}, a_{2}\right\} \rightarrow \infty, b_{j}>a_{j} \geq 0, j=1,2
$$

(cf. (1.2)). Condition (1.3) is equivalent to the joint fulfillment of the two conditions

$$
\int_{a_{1}}^{b_{1}} \int_{0}^{b_{2}} \phi(x, y) d x d y \rightarrow 0 \quad \text { as } b_{1}>a_{1} \rightarrow \infty \text { and } b_{2}>0 \text { is arbitrary, }
$$

and

$$
\int_{0}^{b_{1}} \int_{a_{2}}^{b_{2}} \phi(x, y) d x d y \rightarrow 0 \quad \text { as } b_{2}>a_{2} \rightarrow \infty \text { and } b_{1}>0 \text { is arbitrary. }
$$

It follows immediately that if the double integral (1.1) converges in the regular sense, then it converges in Pringsheim's sense. The converse implication is not true. For example, set

$$
\phi(x, y):= \begin{cases}(-1)^{[x]}[1+x / 2] & \text { for }(x, y) \in[0, \infty) \times[0,1), \\ (-1)^{1+[x]}[1+x / 2] & \text { for }(x, y) \in[0, \infty) \times[1,2), \\ 0 & \text { for }(x, y) \in[2, \infty) \times(2, \infty), \\ \phi(y, x) & \text { for }(x, y) \in[0,2) \times[2, \infty),\end{cases}
$$

where [.] means the integer part of a real number. Since

$$
I\left(\phi ; b_{1}, b_{2}\right)=0 \quad \text { whenever } \min \left\{b_{1}, b_{2}\right\} \geq 2,
$$

the double integral (1.1) converges to zero in Pringsheim's sense. On the other hand, the double integral (1.1) cannot converge in the regular sense, 
since

$$
\int_{2 k-2}^{2 k-1} \int_{0}^{1} \phi(x, y) d x d y=k, \quad k=1,2, \ldots
$$

In the special case when $\phi$ is Lebesgue integrable over the whole quadrant $\overline{\mathbb{R}}_{+}^{2}$, the double integral (1.1) converges in the regular sense, and its limit in Pringsheim's sense is equal to the Lebesgue integral of $\phi$ over $\overline{\mathbb{R}}_{+}^{2}$.

We note that the notions of the 'convergence of a double integral over $\overline{\mathbb{R}}_{+}^{2}$ in Pringsheim's sense or in the regular sense' are the nondiscrete versions of the notions of the 'convergence of a double series $\sum_{k=0}^{\infty} \sum_{\ell=0}^{\infty} a_{k \ell}$ of complex numbers in Pringsheim's sense or in the regular sense', respectively. For details, see [10, Vol. 2, pp. 300-302], 3] and [6].

2. Main results: Uniform convergence of double sine integrals. We investigate the uniform convergence of the family of double sine integrals

$$
\int_{0}^{\infty} \int_{0}^{\infty} f(x, y) \sin u x \sin v y d x d y, \quad(u, v) \in \mathbb{R}_{+}^{2},
$$

where $f: \mathbb{R}_{+}^{2} \rightarrow \mathbb{C}$ is a Lebesgue measurable function.

In order to ensure the existence of the partial integrals

$$
I_{u v}\left(f ; b_{1}, b_{2}\right):=\int_{0}^{b_{1}} \int_{0}^{b_{2}} f(x, y) \sin u x \sin v y d x d y, \quad b_{1}, b_{2}>0
$$

we always assume that for all $\left(b_{1}, b_{2}\right) \in \mathbb{R}_{+}^{2}$,

$$
x y f(x, y) \in L_{\mathrm{loc}}^{1}\left(\overline{\mathbb{R}}_{+}^{2}\right), \quad \text { that is, } \quad \int_{0}^{b_{1}} \int_{0}^{b_{2}} x y|f(x, y)| d x d y<\infty .
$$

We note that the double sine integral (2.1) is the nondiscrete version of the double sine series $\sum_{k=1}^{\infty} \sum_{\ell=1}^{\infty} a_{k \ell} \sin k u \sin \ell v$, where $\left\{a_{k \ell}\right\}$ is a double sequence of complex numbers (for details see [4]). Historically, the first result in this topic is due to Chaundy and Jolliffe [2], who proved the following theorem: If $\left\{a_{k}: k=1,2, \ldots\right\}$ is a decreasing sequence of nonnegative numbers, then the sine series $\sum_{k=1}^{\infty} a_{k} \sin k u$ converges uniformly in $u \in \mathbb{R}_{+}$ if and only if $k a_{k} \rightarrow 0$ as $k \rightarrow \infty$.

In what follows, we always assume that the function $f$ occurring in (2.1) is locally absolutely continuous on $\mathbb{R}_{+}^{2}$, in symbols: $f \in \mathrm{AC}_{\text {loc }}\left(\mathbb{R}_{+}^{2}\right)$, by which we mean the following: the partial derivatives $f_{x}:=\partial f / \partial x$ and $f_{y}:=\partial f / \partial y$ exist everywhere on $\mathbb{R}_{+}^{2}$, and $f$ can be recovered from them in the usual way: 
for all $b_{j}>a_{j}>0, j=1,2$, we have

$$
\begin{aligned}
& \int_{a_{1}}^{b_{1}} f_{x}(x, y) d x=f\left(b_{1}, y\right)-f\left(a_{1}, y\right), \quad y>0, \\
& \int_{a_{2}}^{b_{2}} f_{y}(x, y) d y=f\left(x, b_{2}\right)-f\left(x, a_{2}\right), \quad x>0
\end{aligned}
$$

furthermore, the mixed partial derivatives $f_{x y}, f_{y x}$ exist and $f_{x y}=f_{y x}$ almost everywhere on $\mathbb{R}_{+}^{2}$, and $f_{x}, f_{y}$ can be recovered from them: for all $b_{j}>a_{j}>0, j=1,2$, we have

$$
\begin{aligned}
& \int_{a_{1}}^{b_{1}} f_{x y}(x, y) d x=f_{y}\left(b_{1}, y\right)-f_{y}\left(a_{1}, y\right), \quad y>0, \\
& \int_{a_{2}}^{b_{2}} f_{x y}(x, y) d y=f_{x}\left(x, b_{2}\right)-f_{x}\left(x, a_{2}\right), \quad x>0 .
\end{aligned}
$$

It follows immediately that for all $b_{j}>a_{j}>0, j=1,2$, we have

$$
\int_{a_{1}}^{b_{1}} \int_{a_{2}}^{b_{2}} f_{x y}(x, y) d x d y=f\left(b_{1}, b_{2}\right)-f\left(a_{1}, b_{2}\right)-f\left(b_{1}, a_{2}\right)+f\left(a_{1}, a_{2}\right) .
$$

We refer to [1] and the references in it for the definition and basic properties of absolute continuity of functions in two variables.

Our next definition is motivated by the analogous one in the case of single sine integrals introduced in [7]; the latter was inspired by the corresponding discrete definition in the case of single trigonometric series (see [9]). A function $f \in \mathrm{AC}_{\text {loc }}\left(\mathbb{R}_{+}^{2}\right)$ is said to be of mean value bounded variation, in symbols: $f \in \operatorname{MVBVF}\left(\mathbb{R}_{+}^{2}\right)$, if there exist constants $C$ and $\lambda \geq 2$, depending only on $f$, such that for all $a_{1}, y>0$ we have

$$
\int_{a_{1}}^{2 a_{1}}\left|f_{x}(x, y)\right| d x \leq \frac{C}{a_{1}} \int_{\lambda^{-1} a_{1}}^{\lambda a_{1}}|f(x, y)| d x
$$

for all $x, a_{2}>0$ we have

$$
\int_{a_{2}}^{2 a_{2}}\left|f_{y}(x, y)\right| d y \leq \frac{C}{a_{2}} \int_{\lambda^{-1} a_{2}}^{\lambda a_{2}}|f(x, y)| d y
$$

and for all $a_{1}, a_{2}>0$ we have

$$
\int_{a_{1}}^{2 a_{1}} \int_{a_{2}}^{2 a_{2}}\left|f_{x y}(x, y)\right| d x d y \leq \frac{C}{a_{1} a_{2}} \int_{\lambda^{-1} a_{1}}^{\lambda a_{1}} \int_{\lambda^{-1} a_{2}}^{\lambda a_{2}}|f(x, y)| d x d y .
$$


In our first main result, we give sufficient conditions for the uniform convergence of the double sine integrals (2.1) in the regular sense.

Theorem 1. Assume the function $f: \mathbb{R}_{+}^{2} \rightarrow \mathbb{C}$ satisfies condition (2.3) and belongs to the class $\operatorname{MVBVF}\left(\mathbb{R}_{+}^{2}\right)$. If for all $x, y>0$ we have

$$
x y f(x, y) \rightarrow 0 \quad \text { as } \max \{x, y\} \rightarrow \infty,
$$

and for all $b_{1}, b_{2}>0$ we have

$$
\frac{1}{b_{1} b_{2}} \int_{0}^{b_{1}} \int_{0}^{b_{2}} x y|f(x, y)| d x d y \rightarrow 0 \quad \text { as } \max \left\{b_{1}, b_{2}\right\} \rightarrow \infty
$$

then the double sine integrals (2.1) converge in the regular sense uniformly in $(u, v) \in \mathbb{R}_{+}^{2}$.

We note that if the product $x y f(x, y)$ is bounded on $\mathbb{R}_{+}^{2}$, then condition (2.3) is satisfied and condition (2.11) clearly implies (2.12).

In our next theorem, we show that in case $f(x, y) \geq 0$ condition (2.11) is necessary for the uniform convergence of the double sine integrals (2.1) in the regular sense.

TheOREM 2. Assume the function $f: \mathbb{R}_{+}^{2} \rightarrow \overline{\mathbb{R}}_{+}$satisfies condition (2.3) and belongs to the class $\operatorname{MVBVF}\left(\mathbb{R}_{+}^{2}\right)$. If the double sine integrals (2.1) converge in the regular sense uniformly in $(u, v) \in \mathbb{R}_{+}^{2}$, then condition (2.11) holds true.

The following corollary is an immediate consequence of Theorems 1 and 2 .

Corollary 1. Assume the function $f: \mathbb{R}_{+}^{2} \rightarrow \overline{\mathbb{R}}_{+}$satisfies conditions (2.3) and (2.12), and belongs to the class $\operatorname{MVBVF}\left(\mathbb{R}_{+}^{2}\right)$. Then the double sine integrals (2.1) converge in the regular sense uniformly in $(u, v) \in \mathbb{R}_{+}^{2}$ if and only if condition (2.11) is satisfied.

Our next definition is also motivated by [7] and [8]. A function $f \in$ $\mathrm{AC}_{\text {loc }}\left(\mathbb{R}_{+}^{2}\right)$ is said to be of non-onesided bounded variation, in symbols: $f \in$ $\operatorname{NBVF}\left(\mathbb{R}_{+}^{2}\right)$, if there exists a constant $C$, depending only on $f$, such that

$$
\begin{aligned}
& \int_{a_{1}}^{2 a_{1}}\left|f_{x}(x, y)\right| d x \leq C\left(\left|f\left(a_{1}, y\right)\right|+\left|f\left(2 a_{1}, y\right)\right|\right), \quad a_{1}, y>0, \\
& \int_{a_{2}}^{2 a_{2}}\left|f_{y}(x, y)\right| d y \leq C\left(\left|f\left(x, a_{2}\right)\right|+\left|f\left(x, 2 a_{2}\right)\right|\right), \quad x, a_{2}>0,
\end{aligned}
$$




$$
\begin{aligned}
& \int_{a_{1}}^{2 a_{1}} \int_{a_{2}}^{2 a_{2}}\left|f_{x y}(x, y)\right| d x d y \\
& \leq C\left(\left|f\left(a_{1}, a_{2}\right)\right|+\left|f\left(2 a_{1}, a_{2}\right)\right|+\left|f\left(a_{1}, 2 a_{2}\right)\right|+\left|f\left(2 a_{1}, 2 a_{2}\right)\right|\right), \quad a_{1}, a_{2}>0 .
\end{aligned}
$$

We say that a function $f: \mathbb{R}_{+}^{2} \rightarrow \mathbb{R}$ is monotonically decreasing if it is decreasing in each variable and, in addition, for all $x_{2}>x_{1}>0$ and $y_{2}>$ $y_{1}>0$

$$
f\left(x_{1}, y_{1}\right)-f\left(x_{2}, y_{1}\right)-f\left(x_{1}, y_{2}\right)+f\left(x_{2}, y_{2}\right) \geq 0 .
$$

We note that in the literature there exist other variations of the term 'monotonically decreasing' (see, e.g., [5]).

It is clear that if $f: \mathbb{R}_{+}^{2} \rightarrow \overline{\mathbb{R}}_{+}$belongs to the class $\mathrm{AC}_{\text {loc }}\left(\mathbb{R}_{+}^{2}\right)$, then $f$ is monotonically decreasing if and only if

$f_{x}(x, y) \leq 0, \quad f_{y}(x, y) \leq 0 \quad$ and $\quad f_{x y}(x, y) \geq 0 \quad$ almost everywhere; and in this case $f$ clearly belongs to the class $\operatorname{NBVF}\left(\mathbb{R}_{+}^{2}\right)$.

Our second main result is formulated in the following

TheOREM 3. If the function $f: \mathbb{R}_{+}^{2} \rightarrow \mathbb{C}$ belongs to the class $\operatorname{NBVF}\left(\mathbb{R}_{+}^{2}\right)$, then it also belongs to the class $\operatorname{MVBVF}\left(\mathbb{R}_{+}^{2}\right)$. The converse implication is not true.

The next corollary is an immediate consequence of Corollary 1 and Theorem 3 .

Corollary 2. Assume the function $f \in \mathrm{AC}_{\mathrm{loc}}\left(\mathbb{R}_{+}^{2}\right)$ is monotonically decreasing and satisfies conditions (2.3) and (2.12). Then the double sine integrals (2.1) converge in the regular sense uniformly in $(u, v) \in \mathbb{R}_{+}^{2}$ if and only if condition (2.11) is satisfied.

For example, the function

$$
f(x, y):=(x+1)^{\alpha}(y+1)^{\beta}, \quad-2<\alpha, \beta<-1,
$$

is monotonically decreasing and satisfies each of the conditions $(2.3),(2.11)$ and (2.12). Thus, in this case the double sine integrals (2.1) converge in the regular sense uniformly in $(u, v) \in \mathbb{R}_{+}^{2}$.

We note that Theorems 1-3 and Corollaries 1-2 above may be considered as extensions of the analogous ones in [7, Theorems 2 and 3] from single to double sine integrals.

It is clear that under the conditions of Theorem 1, the double sine integrals (2.1) converge in Pringsheim's sense also uniformly in $(u, v) \in \mathbb{R}_{+}^{2}$. Similarly to the proof of Theorem 2 in Section 4 below, the following theorem can be easily proved, which gives a necessary condition for the uniform convergence of the double sine integrals (2.1) in Pringsheim's sense. 
Theorem 4. Assume the function $f: \mathbb{R}_{+}^{2} \rightarrow \overline{\mathbb{R}}_{+}$satisfies condition (2.3) and belongs to the class $\mathrm{MVBVF}\left(\mathbb{R}_{+}^{2}\right)$. If the double sine integrals (2.1) converge in Pringsheim's sense, or only the remainder integrals (cf. (1.2))

$$
\int_{a_{1}}^{b_{1}} \int_{a_{2}}^{b_{2}} f(x, y) \sin u x \sin v y d x d y, \quad b_{j}>a_{j}>0, j=1,2,
$$

converge to 0 as $\min \left\{a_{1}, a_{2}\right\} \rightarrow \infty$ uniformly in $(u, v) \in \mathbb{R}_{+}^{2}$, then

$$
x y f(x, y) \rightarrow 0 \quad \text { as } \min \{x, y\} \rightarrow \infty .
$$

However, all our attempts have failed so far to modify the steps in the proof of Theorem 1 in order to guarantee the uniform convergence of the double sine integrals (2.1) in Pringsheim's sense for all $(u, v) \in \mathbb{R}_{+}^{2}$ under the conditions of Theorem 1 with (2.11) replaced by the weaker (2.16).

\section{Auxiliary results}

Lemma 1. Assume the function $f: \mathbb{R}_{+}^{2} \rightarrow \mathbb{C}$ satisfies condition (2.3) and belongs to the class $\operatorname{MVBVF}\left(\mathbb{R}_{+}^{2}\right)$. If condition (2.11) is satisfied, then for all $a_{1}, y>0$ we have

$$
a_{1} y \int_{a_{1}}^{\infty}\left|f_{x}(x, y)\right| d x \rightarrow 0 \quad \text { as } \max \left\{a_{1}, y\right\} \rightarrow \infty ;
$$

for all $x, a_{2}>0$ we have

$$
x a_{2} \int_{a_{2}}^{\infty}\left|f_{y}(x, y)\right| d y \rightarrow 0 \quad \text { as } \max \left\{x, a_{2}\right\} \rightarrow \infty ;
$$

and for all $a_{1}, a_{2}>0$ we have

$$
a_{1} a_{2} \int_{a_{1}}^{\infty} \int_{a_{2}}^{\infty}\left|f_{x y}(x, y)\right| d x d y \rightarrow 0 \quad \text { as } \max \left\{a_{1}, a_{2}\right\} \rightarrow \infty .
$$

Proof. By (2.11), for every $\varepsilon>0$ there exists $x_{0}=x_{0}(\varepsilon)>0$ such that for all $x, y>0$,

$$
x y|f(x, y)|<\varepsilon \quad \text { if } \max \{x, y\}>x_{0} .
$$

(i) Let $\max \left\{\lambda^{-1} a_{1}, y\right\}>x_{0}$. By (3.4) and (2.8), we estimate as follows:

$$
a_{1} y \int_{a_{1}}^{\infty}\left|f_{x}(x, y)\right| d x=a_{1} y \sum_{k=0}^{\infty} \int_{2^{k} a_{1}}^{2^{k+1} a_{1}}\left|f_{x}(x, y)\right| d x
$$




$$
\begin{aligned}
& \leq a_{1} y \sum_{k=0}^{\infty} \frac{C}{2^{k} a_{1}} \int_{\lambda^{-1} 2^{k} a_{1}}^{\lambda 2^{k} a_{1}}|f(x, y)| d x \leq C y \sum_{k=0}^{\infty} \frac{1}{2^{k}} \int_{\lambda^{-1} 2^{k} a_{1}}^{\lambda 2^{k} a_{1}} \frac{\varepsilon}{x y} d x \\
& =2 C \varepsilon(\ln \lambda) \sum_{k=0}^{\infty} \frac{1}{2^{k}}=4 C(\ln \lambda) \varepsilon \quad \text { if } \max \left\{\lambda^{-1} a_{1}, y\right\}>x_{0} .
\end{aligned}
$$

Since $\varepsilon>0$ is arbitrary, (3.1) is proved.

(ii) In a similar way, (3.2) is proved by making use of (3.4) and (2.9).

(iii) Let $\max \left\{\lambda^{-1} a_{1}, \lambda^{-1} a_{2}\right\}>x_{0}$. By (3.4) and (2.10),

$$
\begin{aligned}
& a_{1} a_{2} \int_{a_{1}}^{\infty} \int_{a_{2}}^{\infty}\left|f_{x y}(x, y)\right| d x d y \\
& \quad=a_{1} a_{2} \sum_{k=0}^{\infty} \sum_{\ell=0}^{\infty} \int_{2^{k} a_{1}}^{2^{k+1} a_{1}} \int_{2^{\ell} a_{2}}^{2^{\ell+1} a_{2}}\left|f_{x y}(x, y)\right| d x d y \\
& \leq \sum_{k=0}^{\infty} \sum_{\ell=0}^{\infty} \frac{C}{2^{k} 2^{\ell}} \int_{\lambda^{-1} 2^{k} a_{1} \lambda^{-1} 2^{\ell} a_{2}}^{\lambda 2^{k} a_{1}} \int_{\lambda 2^{\ell} a_{2}}|f(x, y)| d x d y \\
& \leq \sum_{k=0}^{\infty} \sum_{\ell=0}^{\infty} \frac{C}{2^{k} 2^{\ell}} \int_{\lambda^{-1} 2^{k} a_{1}}^{\lambda 2^{\ell} a_{2}} \int_{\lambda^{-1} 2^{\ell} a_{2}}^{\infty} \frac{\varepsilon}{x y} d x d y \\
& \quad=4 C \varepsilon(\ln \lambda)^{2} \sum_{k=0}^{\infty} \frac{1}{2^{k}} \sum_{\ell=0}^{\infty} \frac{1}{2^{\ell}}=16 C(\ln \lambda)^{2} \varepsilon \quad \text { if } \max \left\{a_{1}, a_{2}\right\}>\lambda x_{0} .
\end{aligned}
$$

Since $\varepsilon>0$ is arbitrary, (3.3) is proved.

Lemma 2. Assume the function $g: \mathbb{R}_{+} \rightarrow \mathbb{C}$ is locally absolutely continuous on $\mathbb{R}_{+}$and such that $x g(x) \in L_{\text {loc }}^{1}\left(\overline{\mathbb{R}}_{+}\right)$. If there exists a constant $C$ such that for every $a>0$,

$$
\int_{a}^{2 a}\left|g^{\prime}(x)\right| d x \leq C(|g(a)|+|g(2 a)|)
$$

then for every $a>0$,

$$
\int_{a}^{2 a}\left|g^{\prime}(x)\right| d x \leq \frac{4 C}{a} \int_{a / 4}^{4 a}|g(x)| d x .
$$

The proof of Lemma 2 is contained in the proof of [7, Theorem 3].

LEMma 3. Assume the function $f: \mathbb{R}_{+}^{2} \rightarrow \overline{\mathbb{R}}_{+}$belongs to the class $\operatorname{MVBVF}\left(\mathbb{R}_{+}^{2}\right)$. Then for all $a, b>0$ we have 


$$
a b f(a, b) \leq(8 C+2) \int_{a / 2 \lambda}^{\lambda a} \int_{b / 2 \lambda}^{\lambda b} f(x, y) d x d y,
$$

where the constants $C$ and $\lambda$ are from the definition of the class $\operatorname{MVBVF}\left(\mathbb{R}_{+}^{2}\right)$.

Proof. By (2.4), for all $a \leq s \leq 2 a$ and $t>0$ we have

$$
f(s, t)-f(a, t)=\int_{a}^{s} f_{x}(x, t) d x .
$$

Using (2.8) and the fundamental theorem of calculus, we have

$$
\begin{aligned}
f(a, t) & \leq \int_{a}^{s}\left|f_{x}(x, t)\right| d x+f(s, t) \\
& \leq \int_{s / 2}^{s}\left|f_{x}(x, t)\right| d x+f(s, t) \leq \frac{2 C}{s} \int_{s / 2 \lambda}^{\lambda s / 2} f(x, t) d x+f(s, t) \\
& \leq \frac{2 C}{s} \int_{a / 2 \lambda}^{\lambda a} f(x, t) d x+f(s, t), \quad a \leq s \leq 2 a \text { and } t>0 .
\end{aligned}
$$

Integrating both sides of (3.6) with respect to $s$ over the interval $[a, 2 a]$ gives

$$
a f(a, t) \leq 2 C \int_{a / 2 \lambda}^{\lambda a} f(x, t) d x+\int_{a}^{2 a} f(x, t) d x, \quad t>0 .
$$

Making use of (2.5) and (2.9), an analogous argument yields

$$
b f(s, b) \leq 2 C \int_{b / 2 \lambda}^{\lambda b} f(s, y) d y+\int_{b}^{2 b} f(s, y) d y, \quad s>0 .
$$

Next, by making use of (2.6) (or (2.7)) and (2.10), a double version of the above argument gives the following: for all $a \leq s \leq 2 a$ and $b \leq t \leq 2 b$ we have

$$
\begin{aligned}
f(a, b) & =\int_{a b}^{s t} \int_{b y}^{t}(x, y) d x d y+f(a, t)+f(s, b)-f(s, t) \\
& \leq \int_{a b}^{s t}\left|f_{x y}(x, y)\right| d x d y+f(a, t)+f(s, b) \\
& \leq \int_{s / 2}^{s} \int_{t / 2}^{t}\left|f_{x y}(x, y)\right| d x d y+f(a, t)+f(s, b)
\end{aligned}
$$




$$
\begin{aligned}
& \leq \frac{4 C}{s t} \int_{s / 2 \lambda}^{\lambda s / 2} \int_{t / 2 \lambda}^{\lambda t / 2} f(x, y) d x d y+f(a, t)+f(s, b) \\
& \leq \frac{4 C}{a b} \int_{a / 2 \lambda}^{\lambda a} \int_{b / 2 \lambda}^{\lambda b} f(x, y) d x d y+f(a, t)+f(s, b) .
\end{aligned}
$$

Integrating both sides of (3.9) with respect to $s \in[a, 2 a]$ and $t \in[b, 2 b]$, we find that

(3.10) $a b f(a, b) \leq 4 C \int_{a / 2 \lambda}^{\lambda a} \int_{b / 2 \lambda}^{\lambda b} f(x, y) d x d y+a \int_{b}^{2 b} f(a, t) d t+b \int_{a}^{2 a} f(s, b) d s$.

Combining inequalities (3.7), (3.8) and (3.10) yields

$$
\begin{aligned}
a b f(a, b) \leq & 4 C \int_{a / 2 \lambda}^{\lambda a} \int_{b / 2 \lambda}^{\lambda b} f(x, y) d x d y \\
& +2 C \int_{a / 2 \lambda}^{\lambda a} \int_{b}^{2 b} f(x, t) d x d t+\int_{a}^{2 a} \int_{b}^{2 b} f(x, t) d x d t \\
& +2 C \int_{a}^{2 a} \int_{b / 2 \lambda}^{\lambda b} f(s, y) d s d y+\int_{a}^{2 a} \int_{b}^{2 b} f(s, y) d s d y
\end{aligned}
$$

whence (3.5) follows immediately, due to the fact that $\lambda \geq 2$.

\section{Proofs of Theorems 1-4}

Proof of Theorem 1. Let an arbitrary $\varepsilon>0$ be given. By conditions (2.11) and (2.12), there exists $b_{0}=b_{0}(\varepsilon)>0$ such that for all $x, y>0$ we have

$$
x y|f(x, y)|<\varepsilon \quad \text { if } \max \{x, y\}>b_{0},
$$

and for all $b_{1}, b_{2}>0$ we have

$$
\frac{1}{b_{1} b_{2}} \int_{0}^{b_{1}} \int_{0}^{b_{2}} x y|f(x, y)| d x d y<\varepsilon \text { if } \max \left\{b_{1}, b_{2}\right\}>b_{0} .
$$

Furthermore, by (3.1)-(3.3) in Lemma 1, there exists $x_{0}=x_{0}(\varepsilon)>0$ such that

$$
\begin{aligned}
& a_{1} y \int_{a_{1}}^{\infty}\left|f_{x}(x, y)\right| d x<\varepsilon \quad \text { if } \max \left\{a_{1}, y\right\}>x_{0}, \\
& x a_{2} \int_{a_{2}}^{\infty}\left|f_{y}(x, y)\right| d y<\varepsilon \quad \text { if } \max \left\{x, a_{2}\right\}>x_{0},
\end{aligned}
$$




$$
a_{1} a_{2} \int_{a_{1}}^{\infty} \int_{a_{2}}^{\infty}\left|f_{x y}(x, y)\right| d x d y<\varepsilon \quad \text { if } \max \left\{a_{1}, a_{2}\right\}>x_{0},
$$

where $\min \left\{a_{1}, a_{2}, x, y\right\}>0$.

Let $y_{0}:=\max \left\{b_{0}, x_{0}\right\}$. We claim that for all $(u, v) \in \mathbb{R}_{+}^{2}$ we have

$$
\begin{aligned}
\left|I_{u v}\left(f ; a_{1}, b_{1} ; a_{2}, b_{2}\right)\right|:=\left|\int_{a_{1}}^{b_{1}} \int_{a_{2}}^{b_{2}} f(x, y) \sin u x \sin v y d x d y\right|<16 \varepsilon \\
\text { whenever } \max \left\{a_{1}, a_{2}\right\}>y_{0} \text { and } b_{j}>a_{j} \geq 0, j=1,2 .
\end{aligned}
$$

To justify this claim, we will distinguish nine cases (i)-(ix). By Fatou's lemma, we may assume that $a_{1}, a_{2}>0$.

CASE (i): $a_{1}<b_{1} \leq 1 / u$ and $a_{2}<b_{2} \leq 1 / v$. By (4.2), we have

$$
\begin{aligned}
\left|I_{u v}\left(f ; a_{1}, b_{1} ; a_{2}, b_{2}\right)\right| & \leq u v \int_{a_{1}}^{b_{1}} \int_{a_{2}}^{b_{2}} x y|f(x, y)| d x d y \\
& \leq u v \int_{0}^{1 / u} \int_{0}^{1 / v} x y|f(x, y)| d x d y<\varepsilon
\end{aligned}
$$

which is (4.6) with $\varepsilon$ in place of $16 \varepsilon$.

CASE (ii): $a_{1}<b_{1} \leq 1 / u$ and $1 / v \leq a_{2}<b_{2}$. Applying Fubini's theorem, we find that

$$
\begin{aligned}
\left|I_{u v}\left(f ; a_{1}, b_{1} ; a_{2}, b_{2}\right)\right| & =\left|\int_{a_{1}}^{b_{1}}(\sin u x)\left(\int_{a_{2}}^{b_{2}} f(x, y) \sin v y d y\right) d x\right| \\
& \leq \int_{a_{1}}^{b_{1}} u x\left|\int_{a_{2}}^{b_{2}} f(x, y) \sin v y d y\right| d x .
\end{aligned}
$$

Integrating by parts with respect to $y$ gives

$$
\begin{aligned}
\left|\int_{a_{2}}^{b_{2}} f(x, y) \sin v y d y\right| & \\
= & \left|\left[-f(x, y) \frac{\cos v y}{v}\right]_{y=a_{2}}^{b_{2}}+\int_{a_{2}}^{b_{2}} f_{y}(x, y) \frac{\cos v y}{v} d y\right| \\
& \leq \frac{1}{v}\left\{\left|f\left(x, a_{2}\right)\right|+\left|f\left(x, b_{2}\right)\right|+\int_{a_{2}}^{b_{2}}\left|f_{y}(x, y)\right| d y\right\} \\
& \leq a_{2}\left|f\left(x, a_{2}\right)\right|+b_{2}\left|f\left(x, b_{2}\right)+a_{2} \int_{a_{2}}^{b_{2}}\right| f_{y}(x, y) \mid d y .
\end{aligned}
$$

Combining (4.7) and (4.8), it follows from (4.1) and (4.4) that 


$$
\begin{aligned}
\left|I_{u v}\left(f ; a_{1}, b_{1} ; a_{2}, b_{2}\right)\right| & \\
& \leq u \int_{a_{1}}^{b_{1}}\left\{x a_{2}\left|f\left(x, a_{2}\right)\right|+x b_{2}\left|f\left(x, b_{2}\right)\right|+x a_{2} \int_{a_{2}}^{b_{2}}\left|f_{y}(x, y)\right| d y\right\} d x \\
& \leq u \int_{a_{1}}^{b_{1}} 3 \varepsilon d x=3 \varepsilon u\left(b_{1}-a_{1}\right) \leq 3 \varepsilon,
\end{aligned}
$$

which is (4.6) with $3 \varepsilon$ in place of $16 \varepsilon$.

CASE (iii): $a_{1}<b_{1} \leq 1 / u$ and $a_{2}<1 / v<b_{2}$. In view of the decomposition

$$
I_{u v}\left(f ; a_{1}, b_{1} ; a_{2}, b_{2}\right)=\left\{\int_{a_{1}}^{b_{1}} \int_{a_{2}}^{1 / v}+\int_{a_{1}}^{b_{1}} \int_{1 / v}^{b_{2}}\right\} f(x, y) \sin u x \sin v y d x d y
$$

the previous Cases (i) and (ii) give (4.6) with $4 \varepsilon$.

CASE (iv): $1 / u \leq a_{1}<b_{1}$ and $a_{2}<b_{2} \leq 1 / v$. This is the symmetric counterpart of Case (ii), but this time in the proof we use (4.3) instead of (4.4). Thus, we have (4.6) again with $3 \varepsilon$.

$\operatorname{CASE}(\mathrm{v}): 1 / u \leq a_{1}<b_{1}$ and $1 / v \leq a_{2}<b_{2}$. Making use of Fubini's theorem and integrating by parts with respect to $y$ (cf. (4.7) and (4.8)) gives

$$
\begin{aligned}
& I_{u v}\left(f ; a_{1}, b_{1} ; a_{2}, b_{2}\right)=\int_{a_{1}}^{b_{1}}(\sin u x)\left\{f\left(x, a_{2}\right) \frac{\cos v a_{2}}{v}\right. \\
& \left.\quad-f\left(x, b_{2}\right) \frac{\cos v b_{2}}{v}+\int_{a_{2}}^{b_{2}} f_{y}(x, y) \frac{\cos v y}{v} d y\right\} d x \\
& =\frac{1}{v}\left\{\left(\cos v a_{2}\right) \int_{a_{1}}^{b_{1}} f\left(x, a_{2}\right) \sin u x d x-\left(\cos v b_{2}\right) \int_{a_{1}}^{b_{1}} f\left(x, b_{2}\right) \sin u x d x\right. \\
& \left.+\int_{a_{2}}^{b_{2}}(\cos v y)\left(\int_{a_{1}}^{b_{1}} f_{y}(x, y) \sin u x d x\right) d y\right\}=: J_{1}+J_{2}+J_{3}, \quad \text { say. }
\end{aligned}
$$

Integrating by parts with respect to $x$, we obtain

$$
\begin{aligned}
\left|J_{1}\right| & \leq \frac{1}{v}\left|\int_{a_{1}}^{b_{1}} f\left(x, a_{2}\right) \sin u x d x\right| \\
& =\frac{1}{v}\left\{f\left(a_{1}, a_{2}\right) \frac{\cos u a_{1}}{u}-f\left(b_{1}, a_{2}\right) \frac{\cos u b_{1}}{u}+\int_{a_{1}}^{b_{1}} f_{x}\left(x, a_{2}\right) \frac{\cos u x}{u} d x\right\} \\
& \leq \frac{1}{u v}\left\{\left|f\left(a_{1}, a_{2}\right)\right|+\left|f\left(b_{1}, a_{2}\right)\right|+\int_{a_{1}}^{b_{1}}\left|f_{x}\left(x, a_{2}\right)\right| d x\right\}
\end{aligned}
$$


Since this time $1 / u \leq a_{1}$ and $1 / v \leq a_{2}$, it follows from (4.1) and (4.3) that

$$
\left|J_{1}\right| \leq a_{1} a_{2}\left|f\left(a_{1}, a_{2}\right)\right|+b_{1} a_{2}\left|f\left(b_{1}, a_{2}\right)\right|+a_{1} a_{2} \int_{a_{1}}^{b_{1}}\left|f_{x}\left(x, a_{2}\right)\right| d x<3 \varepsilon .
$$

In an analogous way, we conclude that

$$
\begin{aligned}
\left|J_{2}\right| & \leq \frac{1}{v}\left|\int_{a_{1}}^{b_{1}} f\left(x, b_{2}\right) \sin u x d x\right| \\
& \leq a_{1} b_{2}\left|f\left(a_{1}, b_{2}\right)\right|+b_{1} b_{2}\left|f\left(b_{1}, b_{2}\right)\right|+a_{1} b_{2} \int_{a_{1}}^{b_{1}}\left|f_{x}\left(x, b_{2}\right)\right| d x<3 \varepsilon .
\end{aligned}
$$

Finally, applying Fubini's theorem and integrating by parts twice, we find that

$$
\begin{aligned}
& \left|J_{3}\right| \leq \frac{1}{v} \int_{a_{2}}^{b_{2}}\left|\int_{a_{1}}^{b_{1}} f_{y}(x, y) \sin u x d x\right| d y \\
= & \frac{1}{v} \int_{a_{2}}^{b_{2}}\left|\left[-f_{y}(x, y) \frac{\cos u x}{u}\right]_{x=a_{1}}^{b_{1}}+\int_{a_{1}}^{b_{1}} f_{x y}(x, y) \frac{\cos u x}{u} d x\right| d y \\
= & \frac{1}{u v} \int_{a_{2}}^{b_{2}}\left|f_{y}\left(a_{1}, y\right) \cos u a_{1}-f_{y}\left(b_{1}, y\right) \cos u b_{1}+\int_{a_{1}}^{b_{1}} f_{x y}(x, y) \cos u x d x\right| d y \\
\leq & \frac{1}{u v} \int_{a_{2}}^{b_{2}}\left\{\left|f_{y}\left(a_{1}, y\right)\right|+\left|f_{y}\left(b_{1}, y\right)\right|+\int_{a_{1}}^{b_{1}}\left|f_{x y}(x, y)\right| d x\right\} d y \\
\leq & a_{1} a_{2} \int_{a_{2}}^{b_{2}}\left|f_{y}\left(a_{1}, y\right)\right| d y+b_{1} a_{2} \int_{a_{2}}^{b_{2}}\left|f_{y}\left(b_{1}, y\right)\right| d y \\
& +a_{1} a_{2} \int_{a_{1}}^{b_{1}} \int_{a_{2}}^{b_{2}}\left|f_{x y}(x, y)\right| d x d y<3 \varepsilon
\end{aligned}
$$

due to (4.4) and (4.5).

Combining (4.10)-(4.13) gives (4.6) with $9 \varepsilon$.

CASE (vi): $1 / u \leq a_{1}<b_{1}$ and $a_{2}<1 / v<b_{2}$. By the decomposition in Case (iii) (see (4.9)), the previous Cases (iv) and (v) give (4.6) with $12 \varepsilon$.

CASE (vii): $a_{1}<1 / u<b_{1}$ and $a_{2}<b_{2} \leq 1 / v$. This is the symmetric counterpart of Case (iii). Thus, we have (4.6) again with $4 \varepsilon$.

CASE (viii): $a_{1}<1 / u<b_{1}$ and $1 / v \leq a_{2}<b_{2}$. This is the symmetric counterpart of Case (vi). Thus, we have (4.6) again with $12 \varepsilon$. 
CASE (ix): $a_{1}<1 / u<b_{1}$ and $a_{2}<1 / v<b_{2}$. Again by the decomposition in Case (iii), the previous Cases (vii) and (viii) give (4.6) with $16 \varepsilon$ as stated.

To sum up, we have proved (4.6) for all $(u, v) \in \mathbb{R}_{+}^{2}$. Since $\varepsilon>0$ is arbitrary, the proof of Theorem 1 is complete.

Proof of Theorem 2. Given arbitrary $a, b>0$, set

$$
u:=\frac{\pi}{2 \lambda a} \quad \text { and } \quad v:=\frac{\pi}{2 \lambda b},
$$

where $\lambda$ is from the definition of the class $\operatorname{MVBVF}\left(\mathbb{R}_{+}^{2}\right)$. Clearly, for all $x$ in the interval $a / 2 \lambda \leq x \leq \lambda a$ we have

$$
\frac{\pi}{4 \lambda^{2}} \leq u x \leq \frac{\pi}{2}
$$

and analogously, for all $b / 2 \lambda \leq y \leq \lambda b$ we have

$$
\frac{\pi}{4 \lambda^{2}} \leq v y \leq \frac{\pi}{2}
$$

Using the nonnegativity of $f$ and applying Lemma 3 yields

$$
\begin{aligned}
& \int_{a / 2 \lambda}^{\lambda a} \int_{b / 2 \lambda}^{\lambda b} f(x, y) \sin u x \sin v y d x d y \\
& \geq\left(\sin \frac{\pi}{4 \lambda^{2}}\right)^{2} \int_{a / 2 \lambda}^{\lambda} \int_{b / 2 \lambda}^{\lambda b} f(x, y) d x d y \geq \frac{1}{8 C+2}\left(\sin \frac{\pi}{4 \lambda^{2}}\right)^{2} a b f(a, b) .
\end{aligned}
$$

By assumption, (1.3) is satisfied with $\phi(x, y):=f(x, y) \sin u x \sin v y$ uniformly in $(u, v) \in \mathbb{R}_{+}^{2}$. Consequently, the integral on the left-hand side in (4.14) converges to zero as $\max \{a, b\} \rightarrow \infty$. A fortiori, for all $a, b>0$ we have

$$
a b f(a, b) \rightarrow 0 \quad \text { as } \max \{a, b\} \rightarrow \infty .
$$

This proves (2.11). The proof of Theorem 2 is complete.

Proof of Theorem 3. (i) If $f \in \operatorname{NBVF}\left(\mathbb{R}_{+}^{2}\right)$, then conditions (2.13)-(2.15) are satisfied. By (2.15), for all $s, t>0$ we have

$$
\begin{aligned}
& \int_{s / 2}^{2 s} \int_{t / 2}^{2 t}\left|f_{x y}(x, y)\right| d x d y \\
& \quad=\left\{\int_{s / 2}^{s} \int_{t / 2}^{t}+\int_{s}^{2 s} \int_{t / 2}^{t}+\int_{s / 2}^{s} \int_{t}^{2 t}+\int_{s}^{2 s} \int_{t}^{2 t}\right\}\left|f_{x y}(x, y)\right| d x d y
\end{aligned}
$$




$$
\begin{aligned}
\leq\{ & |f(s / 2, t / 2)|+|f(s, t / 2)|+\mid f(s / 2, t||+|f(s, t)| \\
& +|f(s, t / 2)|+|f(2 s, t / 2)|+|f(s, t)|+|f(2 s, t)| \\
& +|f(s / 2, t)|+|f(s, t)|+|f(s / 2,2 t)|+|f(s, 2 t)| \\
& +|f(s, t)|+|f(2 s, t)|+|f(s, 2 t)|+|f(2 s, 2 t)|\} \\
= & C\{|f(s / 2, t / 2)|+2|f(s, t / 2)|+2|f(s / 2, t)|+4|f(s, t)| \\
& +|f(2 s, t / 2)|+|f(s / 2,2 t)|+2|f(2 s, t)|+2|f(s, 2 t)|+|f(2 s, 2 t)|\} \\
=: & C A(s, t), \quad \text { say. }
\end{aligned}
$$

Integrating both sides of this inequality with respect to $s$ and $t$ over $[3 a / 2,2 a]$ and $[3 b / 2,2 b]$, respectively, gives

$$
\begin{aligned}
\int_{3 a / 2}^{2 a} \int_{3 b / 2}^{2 b}\left\{\int_{s / 2}^{2 s} \int_{t / 2}^{2 t}\left|f_{x y}(x, y)\right| d x d y\right\} d s d t & \\
& \leq \int_{3 a / 2}^{2 a} \int_{3 b / 2}^{2 b} A(s, t) d s d t, \quad a, b \geq 0 .
\end{aligned}
$$

Now, we observe that if $3 a / 2 \leq s \leq 2 a$, then $s / 2 \leq a$ and $2 s \geq 3 a$; and analogous inequalities hold for $t$ if $3 b / 2 \leq t \leq 2 b$; that is,

$$
\begin{array}{rlll}
{[s / 2,2 s]} & \supset[a, 2 a] & \text { whenever } & s \in[3 a / 2,2 a], \\
{[t / 2,2 t]} & \supset[b, 2 b] & \text { whenever } & t \in[3 b / 2,2 b] .
\end{array}
$$

Consequently, we conclude that

$$
\begin{aligned}
& \int_{3 a / 2}^{2 a} \int_{3 b / 2}^{2 b}\left\{\int_{s / 2}^{2 s} \int_{t / 2}^{2 t}\left|f_{x y}(x, y)\right| d x d y\right\} d s d t \\
& \geq \int_{3 a / 2}^{2 a} \int_{3 b / 2}^{2 b}\left\{\int_{a}^{2 a} \int_{b}^{2 b}\left|f_{x y}(x, y)\right| d x d y\right\} d s d t=\frac{a b}{4} \int_{a}^{2 a} \int_{b}^{2 b}\left|f_{x y}(x, y)\right| d x d y .
\end{aligned}
$$

On the other hand, by (4.15) we can estimate as follows (integrating by substitution):

$$
\begin{aligned}
\int_{3 a / 2}^{2 a} \int_{3 b / 2}^{2 b} A(s, t) d s d t & \\
= & C\left\{4 \int_{3 a / 4}^{a} \int_{3 b / 4}^{b}\left|f\left(s_{1}, t_{1}\right)\right| d s_{1} d t_{1}+4 \int_{3 a / 2}^{2 a} \int_{3 b / 4}^{b}\left|f\left(s, t_{1}\right)\right| d s d t_{1}\right. \\
& +4 \int_{3 a / 4}^{a} \int_{3 b / 2}^{2 b}\left|f\left(s_{1}, t\right) d s_{1} d t+4 \int_{3 a / 2}^{2 a} \int_{3 b / 2}^{2 b}\right| f(s, t) \mid d s d t
\end{aligned}
$$




$$
\begin{aligned}
& +\int_{3 a}^{4 a} \int_{3 b / 4}^{b}\left|f\left(s_{1}, t_{1}\right)\right| d s_{1} d t_{1}+\int_{3 a / 4}^{a} \int_{3 b}^{4 b}\left|f\left(s_{1}, t_{1}\right)\right| d s_{1} d t_{1} \\
& +\int_{3 a / 2}^{2 a} \int_{3 b}^{4 b}\left|f\left(s, t_{1}\right)\right| d s d t_{1}+\int_{3 a}^{4 a} \int_{3 b / 2}^{2 b}\left|f\left(s_{1}, t\right)\right| d s_{1} d t \\
& \left.+\frac{1}{4} \int_{3 a}^{4 a} \int_{3 b}^{4 b}\left|f\left(s_{1}, t_{1}\right)\right| d s_{1} d t_{1}\right\} \\
& \leq 4 C \int_{3 a / 4}^{4 a} \int_{3 b / 4}^{4 b}|f(s, t)| d s d t
\end{aligned}
$$

where we exploited the fact that the integrals in the braces $\{\cdot\}$ above are over disjoint domains.

Combining (4.16)-(4.18) yields

$$
\frac{a b}{4} \int_{a}^{2 a} \int_{b}^{2 b}\left|f_{x y}(x, y)\right| d x d y \leq 4 C \int_{3 a / 4}^{4 a} \int_{3 b / 4}^{4 b}|f(s, t)| d s d t
$$

which is equivalent to (2.10) with $16 C$ in place of $C$ and $\lambda:=4$.

As to the fulfillment of conditions (2.8) and (2.9), we apply Lemma 2 for $g(x):=f(x, y)$ where $y>0$ is fixed, then for $g(y):=f(x, y)$ where $x>0$ is fixed. As a result, we conclude that (2.8) follows from (2.13), while (2.9) follows from (2.14), with $4 C$ in place of $C$ and $\lambda:=4$ in both cases.

(ii) Define

$$
g(x):=\frac{1}{1+x} \sin \left(\frac{\pi}{\ln 2} \ln x\right), \quad x \in \mathbb{R}_{+} .
$$

Clearly, $g(x) \rightarrow 0$ as $x \rightarrow \infty, x g(x) \in L_{\text {loc }}^{1}\left(\overline{\mathbb{R}}_{+}\right)$, and

$$
g^{\prime}(x)=\frac{\pi}{\ln 2} \frac{1}{x(1+x)} \cos \left(\frac{\pi}{\ln 2} \ln x\right)-\frac{1}{(1+x)^{2}} \sin \left(\frac{\pi}{\ln 2} \ln x\right) .
$$

It follows that $g \in \mathrm{AC}_{\text {loc }}\left(\mathbb{R}_{+}\right)$. Since

$$
g\left(2^{k}\right)=\frac{1}{1+2^{k}} \sin k \pi=0, \quad k=1,2, \ldots,
$$

$g$ cannot belong to the class $\operatorname{NBVF}\left(\mathbb{R}_{+}\right)$.

On the other hand, we claim that $g$ belongs to $\operatorname{MVBVF}\left(\mathbb{R}_{+}\right)$. Indeed, for any $a>0$, an elementary argument gives 


$$
\begin{aligned}
\int_{a}^{2 a}\left|g^{\prime}(x)\right| & d x \\
\leq & \frac{\pi}{\ln 2} \frac{1}{a} \int_{a}^{2 a} \frac{1}{1+x}\left|\cos \left(\frac{\pi}{\ln 2} \ln x\right)\right| d x+\frac{1}{a} \int_{a}^{2 a} \frac{1}{1+x}\left|\sin \left(\frac{\pi}{\ln 2} \ln x\right)\right| d x \\
& =\frac{\pi}{\ln 2} \frac{1}{a} \int_{a}^{2 a} \frac{1}{1+x}\left|\sin \left(\frac{\pi}{\ln 2} \ln x+\frac{\pi}{2}\right)\right| d x+\frac{1}{a} \int_{a}^{2 a}|g(x)| d x \\
& =\frac{\pi}{\ln 2} \frac{1}{a} \int_{\lambda^{-1} a}^{2 \sqrt{2} a} \frac{1}{\sqrt{2}+u}\left|\sin \left(\frac{\pi}{\ln 2} \ln u\right)\right| d u+\frac{1}{a} \int_{a}^{2 a}|g(x)| d x \\
& \leq C \int_{\lambda a}|g(u)| d u, \quad \text { where } u:=\sqrt{2} x, C:=\frac{\pi}{\ln 2}+1, \lambda:=2 \sqrt{2} .
\end{aligned}
$$

Now, define

$$
f(x, y):=g(x) g(y), \quad(x, y) \in \mathbb{R}_{+}^{2} .
$$

It is easy to check that $f \notin \operatorname{NBVF}\left(\mathbb{R}_{+}^{2}\right)$, but $f \in \operatorname{MVBVF}\left(\mathbb{R}_{+}^{2}\right)$.

The proof of Theorem 3 is complete.

Proof of Theorem 4. It runs along the same lines as the proof of Theorem 2 , with the modification that this time $\min \{a, b\} \rightarrow \infty$ (instead of $\max \{a, b\} \rightarrow \infty)$.

Acknowledgements. The authors thank the referee for valuable suggestions to improve the presentation and for calling our attention to paper [5].

\section{References}

[1] E. Berkson and T. A. Gillespie, Absolutely continuous functions for two variables and well-bounded operators, J. London Math. Soc. (2) 30 (1984), 305-324.

[2] T. W. Chaundy and A. E. Jolliffe, The uniform convergence of a certain class of trigonometric series, Proc. London Math. Soc. 15 (1916), 214-216.

[3] G. H. Hardy, On the convergence of certain multiple series, Proc. Cambridge Philos. Soc. 19 (1916-1919), 86-95.

[4] P. Kórus and F. Móricz, On the uniform convergence of double sine series, Studia Math. 193 (2009), 79-97.

[5] W. Lindner, Some properties of the set of discontinuity points of a monotonic function of several variables, Folia Math. 11 (2004), 53-57.

[6] F. Móricz, Some remarks on the notion of regular convergence of multiple series, Acta Math. Hungar. 41 (1983), 161-168.

[7] —, On the uniform convergence of sine integrals, J. Math. Anal. Appl. 354 (2009), 213-219.

[8] D. S. Yu and S. P. Zhou, A generalization of the monotonicity condition and applications, Acta Math. Hungar. 115 (2007), 247-267. 
[9] S. P. Zhou, P. Zhou and D. S. Yu, Ultimate generalization to monotonicity for uniform convergence of trigonometric series, arXiv:math/0611805v1.

[10] A. Zygmund, Trigonometric Series, Cambridge Univ. Press, Cambridge, 1959.

Péter Kórus, Ferenc Móricz

Bolyai Institute

University of Szeged

Aradi vértanúk tere 1

Szeged 6720, Hungary

E-mail: Korus.Peter@stud.u-szeged.hu

moricz@math.u-szeged.hu

Received July 14, 2010

Revised version October 15, 2010 